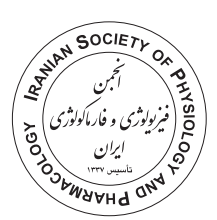

\title{
The cardiovascular responses after lipopolysaccharide microinjection into the dorsomedial periaqueductal gray in rats
}

\author{
Reza Mohebbati ${ }^{1}$, Reza Nejad Shahrokh Abadi $^{2}$, Vida Alikhani ${ }^{3}$, Mohammad Naser Shafei ${ }^{1,3^{*}}$ (iD) \\ 1. Applied Biomedical Research Center, Mashhad University of Medical Sciences, Mashhad, Iran \\ 2. Research Committee, Faculty of Medicine, Mashhad University of Medical Sciences, Mashhad, Iran \\ 3. Department of Physiology, Faculty of Medicine, Mashhad University of Medical Sciences, Mashhad, Iran
}

\begin{abstract}
Introduction: The dorsomedial periaqueductal gray (dmPAG) is located around the cerebral aqueduct with various functions such as cardiovascular regulation and is affected by inflammation. Lipopolysaccharide (LPS) is a complex molecule with an inflammatory effect that is known to affect blood pressure. In the present study, the cardiovascular effect of LPS microinjection into the dmPAG was investigated.

Methods: Rats were divided into three groups consisting of 1: control; 2: $50 \mathrm{ng}$ LPS and 3: $100 \mathrm{ng}$ LPS. Rats were mounted on a stereotaxic device after anesthesia and a continuous recording of cardiovascular parameters was done by a PowerLab device, connected to a cannulated femoral artery and drugs microinjected into dmPAG. The changes $(\Delta)$ in systolic blood pressure (SBP), diastolic blood pressure (DBP), mean arterial pressure (MAP) and heart rate (HR) were calculated at different times and compared to the control group.

Results: Both doses of LPS when microinjected into the dmPAG brought on a significant hypotensive response in the pressure parameters (MAP, SBP, and DBP) when compared to control. A non-significant increase in HR was also documented in both groups.

Conclusion: The results of this experiment indicated that LPS, when microinjected into the dmPAG, induced a hypotensive response in anesthetized rats in both doses in comparison to control.
\end{abstract}

Keywords:

Dorsomedial periaqueductal gray

Lipopolysaccharide

Inflammation

Blood pressure

\section{Introduction}

The periaqueductal gray (PAG) is located around the cerebral aqueduct of the brain and is involved in numerous functions such as stress, defensive reactions and cardiovascular regulation (Bandler and Shipley, 1994). The PAG has connections to several parts of the brain and is functionally divided into four subdivisions:
dlPAG, dmPAG, IPAG and the vlPAG. Although the roles of the dIPAG and the vlPAG subdivisions have been documented in previous studies, such as the hypertensive effect of nitric oxide in the dlPAG (Abadi et al., 2020) or the hypotensive effect of excitatory amino acids in the vlPAG (Keay et al., 1997), few have been done on the dmPAG. The dmPAG receive inputs

\footnotetext{
* Corresponding author: Mohammad Naser Shafei, Shafeimn@mums.ac.ir

Received 8 October 2020; Revised from 30 January 2021; Accepted 14 February 2021
}

Citation: Mohebbati R, Nejad Shahrokh Abadi R, Alikhani V, Shafei MN. The cardiovascular responses after lipopolysaccharide microinjection into the dorsomedial periaqueductal gray in rats. Physiology and Pharmacology 2021; 25:334-340. http://dx.doi.org/10.52547/phypha.25.4.6 
from the different segments of the spinal cord and the ventrolateral medulla (Dampney et al., 2013) and has outputs to the parabrachial complex, the midline medulla and the rostral ventrolateral medulla (RVLM) which contain sympathetic premotor neurons (Dampney et al., 2013). In a previous study, excitatory effects of the glutamatergic system on the cardiovascular system were evaluated in the dmPAG and it was proposed that this effect is mediated by the sympathetic system (Pelosi et al., 2012).

Lipopolysaccharides (LPS) are molecules consisting of lipids and polysaccharides and act as barriers on most Gram-negative bacteria (Bertani and Ruiz, 2018). LPS causes an inflammatory response by acting on its innate receptor, the toll-like receptor 4 (TLR4) (Yücel et al., 2017) and the release of inflammatory cytokines (Frazier et al., 2012; Tong et al., 2020) resulting in hypotension. LPS has also been seen to cause neuroinflammation in acute intraperitoneal injection through the pro-inflammatory cytokines (Gong et al., 2019) and induce expression of angiotensin 2 receptor 1 (AT1) (Li et al., 2015). The renin-angiotensin-system (RAS) also has pro-inflammatory properties along with its main role as a cardiovascular pressure modifier (SA Capettini et al., 2012). The AT1 receptor can cause pro-inflammatory responses when stimulated, especially in the cerebral vasculature (Benicky et al., 2009; Justin et al., 2018). A link between LPS induced hypotension and the preoptic anterior hypothalamic area (POA) (Yilmaz et al., 2008), which projects into the PAG (Simerly and Swanson, 1988) has been found.

In an adult rat brain the AT1 receptor has been found in the nucleus of the solitary tract (NTS) (Song et al., 1992), dorsomedial nuclei of the hypothalamus (DMH), the RVLM, the preoptic region, both the medial and lateral parabrachial nuclei (Lenkei et al., 1997) and the PAG. A previous study has shown that angiotensin injection into the NTS created a dose-dependent response with lower doses causing hypotension (Rettig et al., 1986).

Blood supplied angiotensin 2 has been shown to up-regulate the AT1 receptor in the paraventricular nucleons (Wei et al., 2009). Peripheral injection of LPS has been shown to increase IL-1 $\beta$ in the brain and activate neurons (Konsman et al., 1999). Seeing as how the dmPAG has connections to various cardiovascular control centers in the brain and the relationship between LPS and this system, this paper evaluated the effects of LPS on the cardiovascular system when microinjected into the dmPAG.

\section{Methods and materials}

\section{Animals}

Fifteen male Wistar rats were used in this experiment weighing $250 \pm 20 \mathrm{~g}$. A 12:12-h light-dark cycle environment was used and the animals were given free access to food and water. All experimental procedures were approved by the ethical committee of Mashhad University of Medical Sciences (IR.MUMS.MEDICAL. REC.1397.029).

\section{Drugs}

The drugs used in this study were urethane and LPS. Both drugs were procured from Sigma Company, USA.

\section{Experiment groups}

Rats randomly were divided into three groups, as follows ( $\mathrm{n}=5), 1$ : control group, microinjection of saline into the PAG; 2: LPS 50, microinjection of 50ng LPS into the PAG and 3: LPS 100, microinjection of 100ng LPS into the PAG. LPS (50 and 100ng, Gao et al., 1999) was weighted and then it was dissolved in 100nl saline and microinjected.

\section{Experiment protocol}

An intraperitoneal injection of urethane $(1.4 \mathrm{~g} / \mathrm{kg}$, IP) was used to anesthetize the rats (Shafei and Nasimi, 2011). We used urethane because of safety and fewer effects on blood pressure. The PowerLab system (AD Instruments, Bella Vista, NSW, Australia) was used to record the cardiovascular parameters via a blood pressure transducer, which was connected to an angiocath inserted into the left femoral artery (Mohebbati et al., 2019). The rats were then mounted onto a stereotaxic device, their skull was exposed and a hole drilled over the dmPAG (AP: 7.3mm, L: 0mm, H: 4.9mm) (Paxinos and Watson, 2006). Cardiovascular responses including heart rate (HR), mean arterial pressure (MAP) and systolic/diastolic blood pressure (SBP/DBP) were recorded after 10min of stabilization. When the parameters stabilized, saline and the two doses of LPS were microinjected, using a manual injector connected to a single-barreled micropipette, individually into the dmPAG and with subsequent responses recorded for 20min after, and the peak change $(\Delta)$ was documented. 


\section{Statistical analysis}

Cardiovascular parameters before and after microinjection of LPS into the dmPAG were recorded and changes $(\Delta$, differences between post and pre-injection) in systolic blood pressure $(\triangle \mathrm{SBP})$, diastolic blood pressure $(\triangle \mathrm{DBP})$, means arterial pressure $(\triangle \mathrm{MAP})$ and heart rate $(\triangle \mathrm{HR})$ were calculated, with the data being expressed as mean \pm SEM. Data normality was checked using the Kolmogroph Smirnov test. Statistical analysis was done by One-Way ANOVA with post hock Tuckey between groups and we used paired T-test for time comparisons (GraphPad, InStat version 3.10). The P-value of $<0.05$ being used to determine the significance.

\section{Results}

Microinjection of saline into the dmPAG and its cardiovascular response

After the stabilizing period, saline was microinjected into the dmPAG and changes in parameters were obtained and have been shown in Table 1. There were no significant changes in all parameters when compared to pre-injection $(P>0.05)$.

Microinjection of two doses of LPS (50 and 100ng) into the dmPAG and its cardiovascular response

Microinjection of the lower dose of LPS (50ng) into the dmPAG increased $\triangle \mathrm{HR}$ but this effect was not significant $(P>0.05)$ when compared to changes induced by the saline group. This dose of LPS significantly decreased

TABLE 1: The comparison between cardiovascular parameters in the saline group over time. Data are presented as mean \pm SEM. HR: heart rate, SBP: systolic blood pressure, DBP: diastolic blood pressure, MAP: mean arterial pressure

\begin{tabular}{|lcccc|}
\multicolumn{1}{c}{ Cardiovascular parameters } & HR & SBP & DBP & MAP \\
Time & $($ Beat $/ \mathrm{min})$ & $(\mathrm{mmHg})$ & $(\mathrm{mmHg})$ & $(\mathrm{mmHg})$ \\
Before injection & $340.9 \pm 7.2$ & $141 \pm 2.9$ & $110 \pm 6.4$ & $119 \pm 4.2$ \\
After injection & $348 \pm 6.1$ & $145 \pm 3.6$ & $115 \pm 5.2$ & $120 \pm 4.9$
\end{tabular}

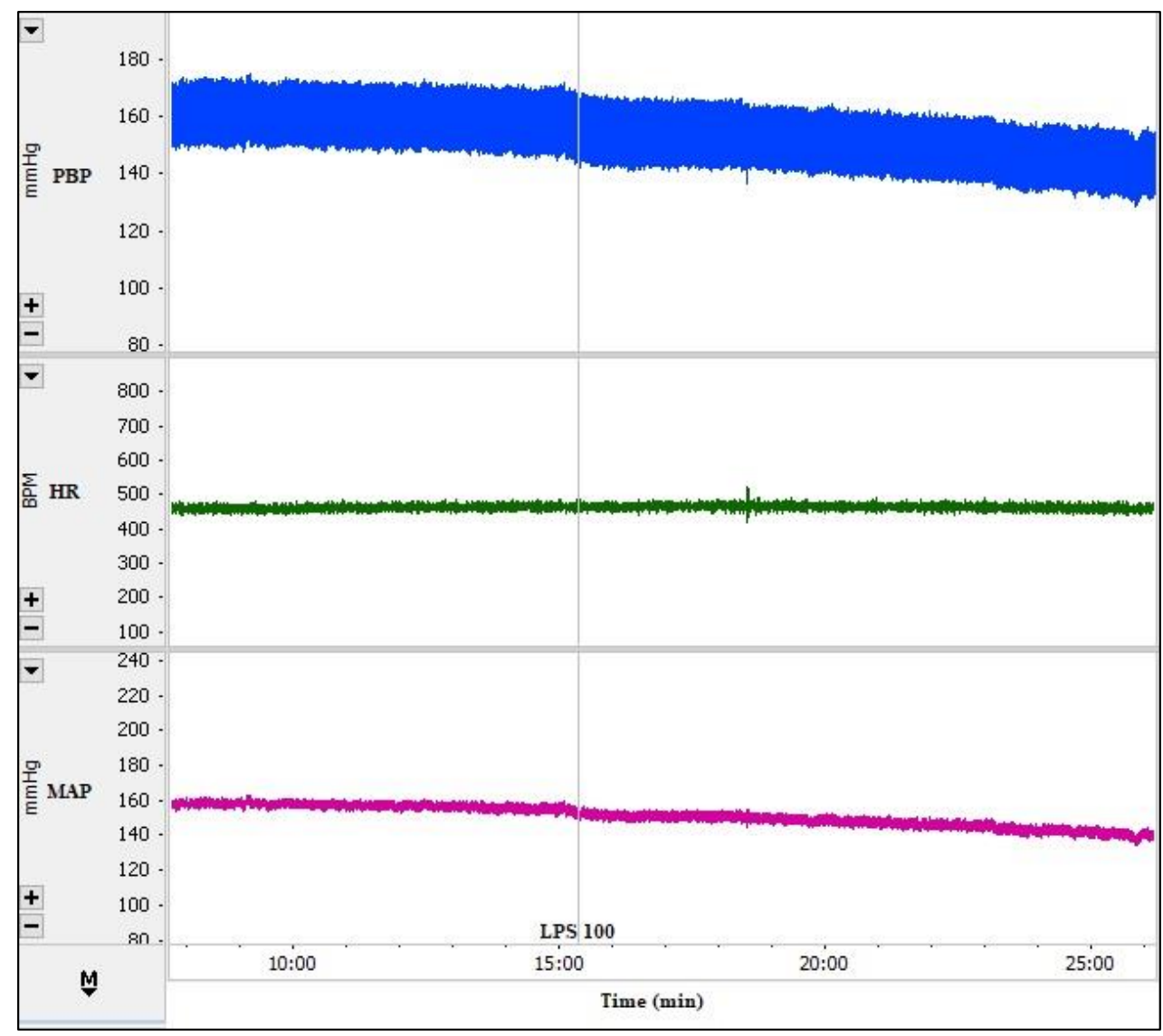

FIGURE 1. A recorded sample for changes of cardiovascular parameters after microinjection of the LPS into the dmPAG in rats. PBP: pulsatile blood pressure, HR: heart rate, MAP: mean arterial pressure 


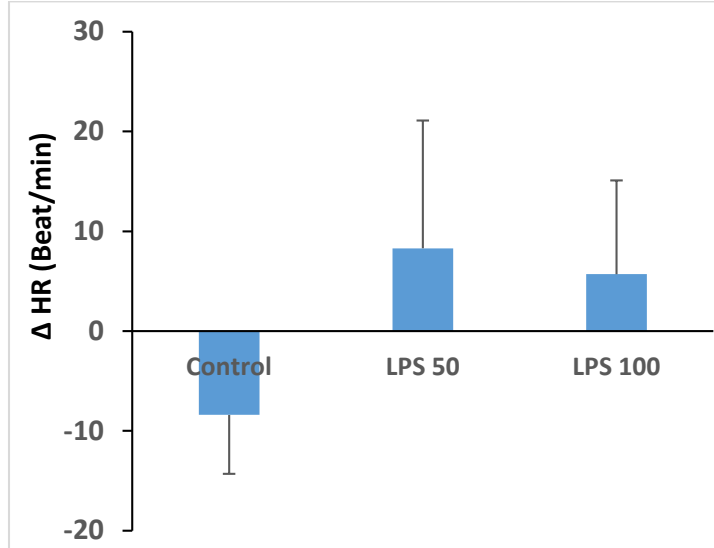

FIGURE 2. Peak changes of heart rate $(\triangle \mathrm{HR})$ after microinjection of the LPS into the dmPAG in rats. The data are expressed as mean \pm SEM; $n=5$.

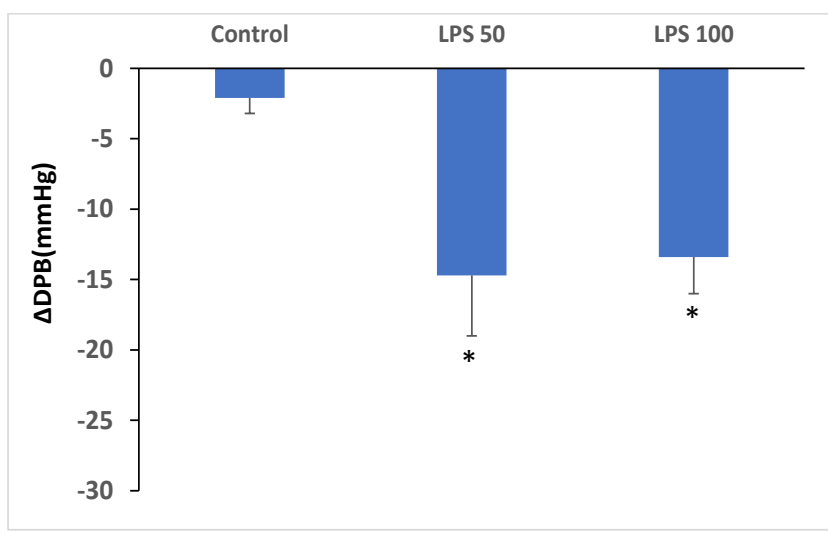

FIGURE 4. Peak changes of diastolic blood pressure $(\triangle \mathrm{DBP})$ after microinjection of the LPS into the dmPAG in rats. The data are expressed as mean \pm SEM; $\mathrm{n}=5 .{ }^{*} P<0.05$ compared to the control.

changes in blood pressure parameters $(\triangle \mathrm{SBP}, \triangle \mathrm{DBP}$ and $\triangle \mathrm{MAP})$ compared to the control group $(P<0.05$ for all responses). Microinjection of the higher dose (100ng) of LPS into the dmPAG also non-significantly increased $\Delta$ HR compared to control. Also, we found no significant differences between the $\triangle \mathrm{HR}$ in two doses of LPS. The higher dose also decreased changes in blood pressure parameters $(\triangle \mathrm{SBP}, \triangle \mathrm{DBP}$ and $\triangle \mathrm{MAP})$ compared to saline $(P<0.05$ for all parameters). Comparison of changes in the blood pressure parameters in the two doses also did not show a significant difference (Figures $1-5)$.

\section{Discussion}

The results showed that both doses of LPS when microinjected into the dmPAG significantly decreased all blood pressure parameters while non-significantly increased HR. This study indicates that LPS has a

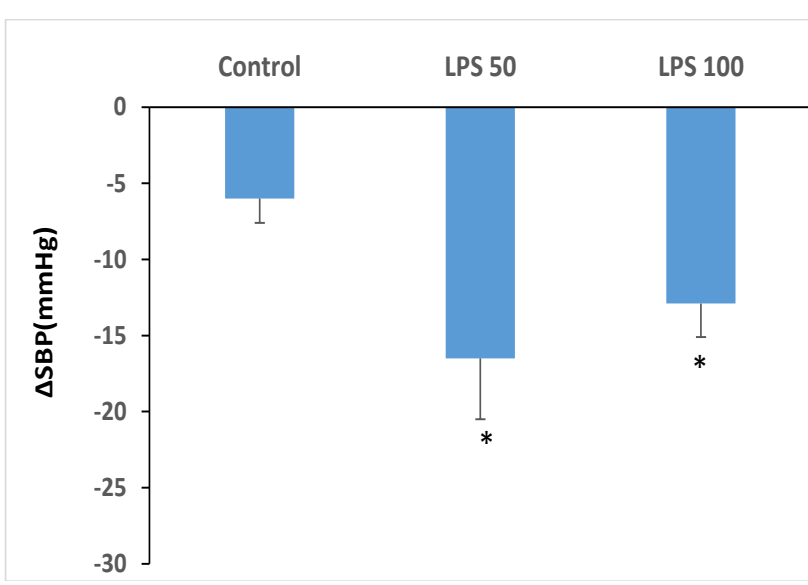

FIGURE 3. Peak changes of systolic blood pressure $(\triangle \mathrm{SBP})$ after microinjection of the LPS into the dmPAG in rats. The data are expressed as mean $\pm \mathrm{SEM} ; \mathrm{n}=5 .{ }^{*} P<0.05$ compared to the control.

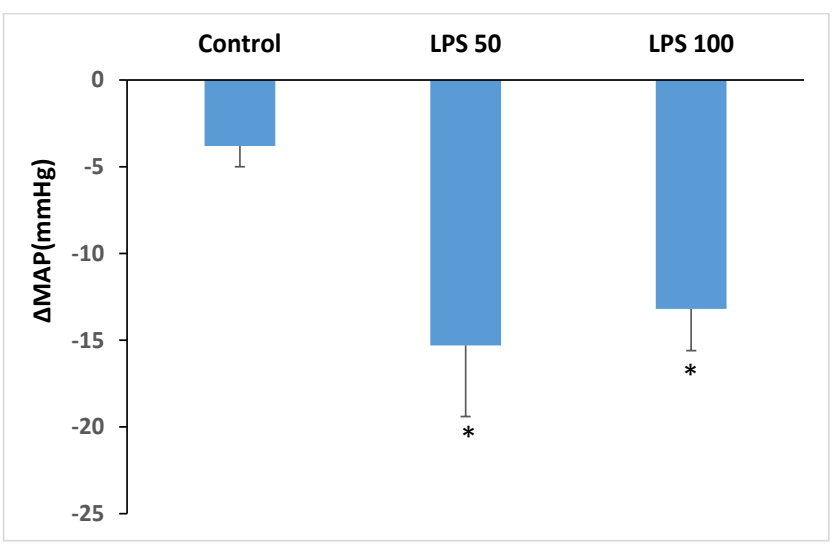

FIGURE 5. Peak changes of mean arterial pressure ( $\triangle \mathrm{MAP})$ after microinjection of the LPS into the dmPAG in rats. The data are expressed as mean \pm SEM; $\mathrm{n}=5$. ${ }^{*} P<0.05$ compared to the control.

cardiovascular effect in the dmPAG in line with a previous study (Doyle et al., 2017). Our results also are similar to intravenous injection shows a hypotensive effect (Temiz-Resitoglu et al., 2017; Yilmaz et al., 2008). The mechanisms for the hypotensive response from LPS in the dmPAG are unknown, but this area has a projection to several brain areas involve in cardiovascular regulation. The two main of these projections are to the RVLM and the parabrachial complex.

Microinjection of LPS into the dmPAG activated the microglia in this nucleus (Sugama et al., 2009) through their TLR4 (Lehnardt et al., 2003), which leads to the activation of the NF- $\mathrm{kB}$ and mitogen-activated protein kinases pathway and the release of inflammatory cytokines like IL-1 $\beta$ (Frazier et al., 2012; Tong et al., 2020). A previous study showed increased expression of IL- $1 \beta$ in the PAG and increased immunoreactivity in the with LPS induced inflammation of the vocal cords 
(Simonyan et al., 2012). Neuroinflammation, via upregulation of iNOS and cyclooxygenase 2 expression and increased pro-inflammatory cytokines IL-1 $\beta$ and IL-6, can be seen in intraperitoneal injection of LPS (Gong et al., 2019).

LPS can also induce expression of AT1 (Li et al., 2015) and stimulate angiotensin II production (Gong et al., 2019; Zhang and Sun, 2005), with the induction of the AT1 receptor being seen in the rat pituitary gland after systemic administration of LPS (Sánchez-Lemus et al., 2009). The RAS also has pro-inflammatory properties along with its main role as a cardiovascular pressure modifier (Sa Capettini et al., 2012). Angiotensin can also act on brain microglia and increase cytokine and reactive oxygen species production (Biancardi et al., 2017) through the NADPH enzyme. Local tissue RAS components have also been found in the CNS (Paul et al., 2006).

The AT1 receptor can cause pro-inflammatory responses when stimulated, especially in the cerebral vasculature (Benicky et al., 2009; Justin et al., 2018). In an adult rat brain, the AT1 receptor has been found the median preoptic nucleus, the area postrema, the nucleus of the NTS (Song et al., 1992), the paraventricular and $\mathrm{DMH}$, the dorsal raphe nucleus, the RVLM, the preoptic region and both the medial and lateral parabrachial nuclei (Lenkei et al., 1997). The PAG also expresses receptors for angiotensin II and contains both AT1 and AT2 mRNA expression. Studies have shown that the initial systemic LPS endotoxin caused hypotension is in part mediated by the POA (Yilmaz et al., 2008). The POA projects into the PAG (Simerly and Swanson, 1988), with the hypotension induced by its activation being attenuated by destruction (Inui et al., 1995) or inhibition (Behbehani and Da Costa Gomez, 1996) of neurons in the vlPAG. A study has shown that angiotensin injection into the NTS created a dose-dependent response with lower doses causing hypotension (Rettig et al., 1986). A previous study has shown that continuous treatment of PAG neurons with LPS increased ion current induced by glycine (an inhibitory neurotransmitter in the brainstem) while reducing that of the excitatory glutamate (Shin et al., 2003).

The RVLM contains sympathetic premotor neurons and causes a presser response when stimulated (Kiely and Gordon, 1994). A previous study showed stimulation of the dmPAG brought on a pressor response (Pelosi et al., 2012). The RVLM is also partly responsible for hypertension induced by systemic inflammation, through neuro-inflammation and oxidative stress ( $\mathrm{Wu}$ et al., 2012). Although LPS also induces inflammation through cytokines, its effects may be due to inhibition of projections from the dmPAG to the RVLM, possibly through the AT1 receptor. The other region the dmPAG projects to is the parabrachial complex. Both electrical and chemical stimulation in this nucleus through glutamate (an excitatory neurotransmitter) have been shown to elicit a pressor-tachycardia effect (Chamberlin and Saper, 1992). Similar to the RVLM, the effects of LPS microinjection and subsequent hypotension may be a result of inhibition of the dmPAG projections into the parabrachial complex through the AT1 receptor.

Although the exact mechanism of microinjection of LPS into the dmPAG is not known, subsequent inflammation in the vlPAG should not be ruled out. The vlPAG also projects into the RVLM (Dampney et al., 2013), with these outputs being inhibitory (Bowman et al., 2013). More studies need to be done on this matter to gain a clearer picture as no conclusion can be derived on the exact mechanism of the hypotensive effect. Therefore, we suggest the researcher study the ECG and molecular assessments for evaluation of this effect.

\section{Conclusion}

A depressor effect was elicited in both doses of LPS when microinjected into the dorsomedial subdivision of the periaqueductal grey matter. This effect was statistically significant when compared to a control group of saline.

\section{Acknowledgment}

we would like to thank the Research Council of Mashhad University of Medical Sciences for their financial support.

\section{Conflicts of interest}

There are no conflicts of interest.

\section{References}

Abadi RNS, Zangouei AS, Mohebbati R, Shafei MN. Determining the cardiovascular effects of nitric oxide in the dorsolateral Periaqueductal Gray (dlPAG) in anaesthetised rats. J Taibah Univ Medical Sci 2020; 15: 502-8. https://doi. org/10.1016/j.jtumed.2020.10.004 
Bandler R, Shipley MT. Columnar organization in the midbrain periaqueductal gray: modules for emotional expression? Trends Neurosci 1994; 17: 379-89. https://doi. org/10.1016/0166-2236(94)90047-7

Behbehani MM, Gomez TM. Properties of a projection pathway from the medial preoptic nucleus to the midbrain periaqueductal gray of the rat and its role in the regulation of cardiovascular function. Brain Res 1996; 740: 141-50. https://doi.org/10.1016/S0006-8993(96)00858-X

Benicky J, Sánchez-Lemus E, Pavel J, Saavedra JM. Anti-inflammatory effects of angiotensin receptor blockers in the brain and the periphery. Cell Mol Neurobiol 2009; 29: 78192. https://doi.org/10.1007/s10571-009-9368-4

Bertani B, Ruiz N. Function and biogenesis of lipopolysaccharides. EcoSal Plus 2018; 8. https://doi.org/10.1128/ecosalplus.ESP-0001-2018

Biancardi VC, Bomfim GF, Reis WL, Al-Gassimi S, Nunes KP. The interplay between angiotensin II, TLR4 and hypertension. Pharmacol Res 2017; 120: 88-96. https://doi. org/10.1016/j.phrs.2017.03.017

Bowman BR, Kumar NN, Hassan SF, Mcmullan S, Goodchild AK. Brain sources of inhibitory input to the rat rostral ventrolateral medulla. J Comp Neurol 2013; 521: 213-32. https://doi.org/10.1002/cne.23175

Chamberlin NL, Saper CB. Topographic organization of cardiovascular responses to electrical and glutamate microstimulation of the parabrachial nucleus in the rat. $\mathrm{J}$ Comp Neurol 1992; 326: 245-62. https://doi.org/10.1002/ cne.903260207

Dampney RA, Furlong TM, Horiuchi J, Iigaya K. Role of dorsolateral periaqueductal grey in the coordinated regulation of cardiovascular and respiratory function. Auton Neurosci 2013; 175: 17-25. https://doi.org/10.1016/j.autneu.2012.12.008

Doyle HH, Eidson LN, Sinkiewicz DM, Murphy AZ. Sex Differences in microglia activity within the periaqueductal gray of the rat: a potential mechanism driving the dimorphic effects of morphine. J Neurosci 2017; 37: 3202-14. https://doi.org/10.1523/JNEUROSCI.2906-16.2017

Frazier WJ, Xue J, Luce WA, Liu Y. MAPK signaling drives inflammation in lps-stimulated cardiomyocytes: the route of crosstalk to G-protein-coupled receptors. Plos One 2012; 7. https://doi.org/10.1371/journal.pone.0050071

Gao N, Wang AJ, Yang Yz, Hu MX, Xie H. Opioid receptor mediated modulation of intrahippocampal enkephalin induced cellular immune function. Sheng Li Xue Bao 1999; 51: 106-10.
Gong X, Hu H, Qiao Y, Xu P, Yang M, Dang R, et al. The involvement of renin-angiotensin system in lipopolysaccharide-induced behavioral changes, neuroinflammation, and disturbed insulin signaling. Front Pharmacol 2019; 10: 318. https://doi.org/10.3389/fphar.2019.00318

Inui K, Nomura J, Murase S, Nosaka S. Facilitation of the arterial baroreflex by the preoptic area in anaesthetized rats. J physiol 1995; 488: 521-31. https://doi.org/10.1113/jphysiol.1995.sp020987

Justin A, Divakar S, Ramanathan M. Cerebral ischemia induced inflammatory response and altered glutaminergic function mediated through brain AT(1) and not AT(2) receptor. Biomed pharmacother 2018; 102: 947-58. https:// doi.org/10.1016/j.biopha.2018.03.164

Keay KA, Crowfoot LJ, Floyd NS, Henderson LA, Christie MJ, Bandler R. Cardiovascular effects of microinjections of opioid agonists into theDepressor Region'of the ventrolateral periaqueductal gray region. Brain Res 1997; 762: 6171. https://doi.org/10.1016/S0006-8993(97)00285-0

Kiely JM, Gordon FJ. Role of rostral ventrolateral medulla in centrally mediated pressor responses. Am J Physiol Heart Circ Physiol 1994; 267. https://doi.org/10.1152/ ajpheart.1994.267.4.H1549

Konsman JP, Kelley K, Dantzer R. Temporal and spatial relationships between lipopolysaccharide-induced expression of fos, interleukin-1 $\beta$ and inducible nitric oxide synthase in rat brain. Neuroscience 1999; 89: 535-48. https://doi. org/10.1016/S0306-4522(98)00368-6

Lehnardt S, Massillon L, Follett P, Jensen FE, Ratan R, Rosenberg PA, et al. Activation of innate immunity in the CNS triggers neurodegeneration through a Toll-like receptor 4-dependent pathway. Proc Natl Acad Sci U S A 2003; 100: 8514-9. https://doi.org/10.1073/pnas.1432609100

Lenkei Z, Palkovits M, Corvol P, Llorens-Cortès C. Expression of angiotensin type-1 (AT1) and type-2 (AT2) receptor mRNAs in the adult rat brain: a functional neuroanatomical review. Frontiers Neuroendocrinol 1997; 18: 383-439. https://doi.org/10.1006/frne.1997.0155

Li HP, Qiu HB, Wang HQ. Effect of lipopolysaccharide on angiotensin II type 1 receptor expression and function in human pulmonary microvascular endothelial cells. Mol Med Rep 2015; 12: 8289-93. https://doi.org/10.3892/ mmr.2015.4481

Mohebbati R, Hosseini M, Khazaei M, Khajavirad A, Shafei MN. The effects of inactivation of pedunculopontine tegmental nucleus by cobalt (II) chloride on cardiovascular responses in hemorrhagic hypotensive rats. 
Basic Clin Neurosci 2019; 10: 235. https://doi.org/10.32598/ bcn.9.10.315

Paul M, Poyan Mehr A, Kreutz R. Physiology of local renin-angiotensin systems. Physiol Rev 2006; 86: 747-803. https://doi.org/10.1152/physrev.00036.2005

Paxinos G, Watson C. The rat brain in stereotaxic coordinates: hard cover edition, 2006.

Pelosi GG, Busnardo C, Tavares RF, Corrêa FMA. Cardiovascular responses to glutamate microinjection in the dorsomedial periaqueductal gray of unanesthetized rats. J Neurosci Res 2012; 90: 2193-200. https://doi.org/10.1002/ jnr.23094

Rettig R, Healy DP, Printz MP. Cardiovascular effects of microinjections of angiotensin II into the nucleus tractus solitarii. Brain Res 1986; 364: 233-40. https://doi. org/10.1016/0006-8993(86)90835-8

SA Capettini L, Montecucco F, Mach F, Stergiopulos N, As Santos R, F Da Silva R. Role of renin-angiotensin system in inflammation, immunity and aging. Curr Pharm Des 2012; 18: 963-70. https://doi.org/10.2174/138161212799436593

Sánchez-Lemus E, Benicky J, Pavel J, Saavedra JM. In vivo Angiotensin II AT1 receptor blockade selectively inhibits LPS-induced innate immune response and ACTH release in rat pituitary gland. Brain Behav Immun 2009; 23: 945-57. https://doi.org/10.1016/j.bbi.2009.04.012

Shafei MN, Nasimi A. Effect of glutamate stimulation of the cuneiform nucleus on cardiovascular regulation in anesthetized rats: role of the pontine kolliker-fuse nucleus. Brain Res 2011; 1385: 135-43. https://doi.org/10.1016/j.brainres.2011.02.046

Shin MC, Jang MH, Chang HK, Kim YJ, Kim EH, Kim CJ. Modulation of cyclooxygenase-2 on glycine- and glutamate-induced ion currents in rat periaqueductal gray neurons. Brain Res Bull 2003; 59: 251-6. https://doi. org/10.1016/S0361-9230(02)00872-9

Simerly RB, Swanson LW. Projections of the medial preoptic nucleus: a Phaseolus vulgaris leucoagglutinin anterograde tract-tracingstudyintherat.JCompNeurol 1988;270:209-42. https://doi.org/10.1002/cne.902700205

Simonyan K, Feng X, Henriquez V, Ludlow C. Combined laryngeal inflammation and trauma mediate long-lasting immunoreactivity response in the brainstem sensory nuclei in the rat. Front Integr Neurosci 2012; 6: 97. https://doi. org/10.3389/fnint.2012.00097

Song K, Allen AM, Paxinos G, Mendelsohn Fa. Mapping of angiotensin II receptor subtype heterogeneity in rat brain. J Comp Neurol 1992; 316: 467-84. https://doi.org/10.1002/ cne.903160407

Sugama S, Takenouchi T, Fujita M, Conti B, Hashimoto $\mathrm{M}$. Differential microglial activation between acute stress and lipopolysaccharide treatment. J Neuroimmunol 2009; 207: 24-31. https://doi.org/10.1016/j.jneuroim.2008.11.007

Temiz-Resitoglu M, Kucukkavruk SP, Guden DS, Cecen P, Sari AN, Tunctan B, et al. Activation of mTOR/I $\kappa \mathrm{B}-\alpha / \mathrm{NF}$ $\kappa \mathrm{B}$ pathway contributes to LPS-induced hypotension and inflammation in rats. Eur J Pharmacol 2017; 802: 7-19. https://doi.org/10.1016/j.ejphar.2017.02.034

Tong W, Chen X, Song X, Chen Y, Jia R, Zou Y, et al. Resveratrol inhibits LPS-induced inflammation through suppressing the signaling cascades of TLR4-NF-אB/MAPKs/IRF3. Exp Ther Med 2020; 19: 1824-34. https://doi.org/10.3892/ etm.2019.8396

Wei SG, Yu Y, Zhang ZH, Felder RB. Angiotensin II upregulates hypothalamic AT1 receptor expression in rats via the mitogen-activated protein kinase pathway. Am J Physiol Heart Circ Physiol 2009; 296. https://doi.org/10.1152/ ajpheart.00942.2008

Wu KL, Chan SHH, Chan JY. Neuroinflammation and oxidative stress in rostral ventrolateral medulla contribute to neurogenic hypertension induced by systemic inflammation. J Neuroinflammation 2012; 9: 1-5. https://doi. org/10.1186/1742-2094-9-212

Yilmaz MS, Millington WR, Feleder C. The preoptic anterior hypothalamic area mediates initiation of the hypotensive response induced by LPS in male rats. Shock 2008; 29: 2327. https://doi.org/10.1097/shk.0b013e3180caac7e

Yücel G, Zhao Z, El-Battrawy I, Lan H, Lang S, Li X, et al. Lipopolysaccharides induced inflammatory responses and electrophysiological dysfunctions in human-induced pluripotent stem cell derived cardiomyocytes. Sci Rep 2017; 7: 1-3. https://doi.org/10.1038/s41598-017-03147-4

Zhang H, Sun GY. LPS induces permeability injury in lung microvascular endothelium via AT1 receptor. Arch Biochem Biophys 2005; 441: 75-83. https://doi.org/10.1016/j. abb.2005.06.022 